\title{
Gram Positive Bacterial Lipoteichoic Acid Role in a Root Canal Infection - A Literature Review
}

\author{
Vinoo Subramaniam Ramachandran ${ }^{1 *}$ (D), Mensudar Rathakrishnan ${ }^{2}$ iD, \\ Malathy Balaraman Ravindrran ${ }^{3}$ D , Alargarsamy Venkatesh ${ }^{2}$ D , Vidhya Shankari \\ Shanmugasundaram ${ }^{4}$ iD and Karpagavinayagam Kumaraguru ${ }^{5}$ (i)
}
${ }^{1}$ Bharath Institute of Higher Education and Research, Agharam Road, Selaiyur, Chennai - 600073 Tamilnadu, India.
${ }^{2}$ Department of Conservative Dentistry and Endodontics, Sree Balaji Dental College and Hospital, Pallikaranai, Chennai - 600 100, Tamilnadu, India.
${ }^{3}$ Department of Microbiology, Sathyabama Dental College and Hospital, Chennai - 600119 ,
Tamilnadu, India.
${ }^{4}$ Department of Conservative Dentistry and Endodontics, Asan Memorial Dental College and Hospital, Asan Nagar, Chengalpattu - 603 105, Tamil Nadu, India.
${ }^{5}$ Private Practice, K \& V Dental Care, Chennai - 600 126, Tamilnadu, India.

\begin{abstract}
Bacteria and its by-products are found to be the main cause of pulpal and periapical infection of tooth. Infected root canals of tooth harbours a wide variation of microbial flora that includes both Gram-positive and Gram-negative microorganisms. Bacterial components such as Lipopolysaccharide (LPS) of gram negative bacteria and Lipoteichoic Acid (LTA) of gram positive bacteria have the potential to enter the peri-apical tissue of tooth and initiate the inflammatory process. After microbial death that occurs either due to body's defence cells or by antibiotic action, bacterial cell wall components such as LTA are released which can persist inside macrophages for prolonged periods causing chronic inflammation. Once these cell-wall components are recognized by the body immune surveillance cells, numerous inflammatory mediators are released leading to inflammation and subsequent pathological consequences. The purpose of this review is intend to summarize the role of gram positive bacterial component LTA in causing endodontic infection and use of potential therapeutic agents against LTA.
\end{abstract}

Keywords: Lipoteichoic acid (LTA), endodontic infection, Enterococcus faecalis, lipoteichoic acid

*Correspondence: vinoomds@yahoo.com

(Received: December 25, 2020; accepted: April 19, 2021)

Citation: Ramachandran VS, Rathakrishnan M, Ravindrran MB, Venkatesh A, Shanmugasundaram VS, Kumaraguru K. Gram Positive Bacterial Lipoteichoic Acid Role in a Root Canal Infection - A Literature Review. J Pure Appl Microbiol. 2021;15(2):534543. doi: 10.22207/JPAM.15.2.29

(C) The Author(s) 2021. Open Access. This article is distributed under the terms of the Creative Commons Attribution 4.0 International License which permits unrestricted use, sharing, distribution, and reproduction in any medium, provided you give appropriate credit to the original author(s) and the source, provide a link to the Creative Commons license, and indicate if changes were made. 


\section{INTRODUCTION}

Evidence based treatment is the new paradigm in global health care. A wide variation of microbial flora is normally present in infected root canals of tooth. A thorough understanding of the microbiota associated with pulp pathology is the basis for the success of endodontic treatment. The microbiota involved in endodontic treatment failure are facultative anaerobes such as Enterococcus, Actinomyces species and Candida. Gram positive Enterococcus faecalis (E.faecalis) is commonly found in high percentage in sites exhibiting root canal failures and it survives as a single organism or as a major component of the flora in the root canals of tooth. Bacterial virulence factors which are primarily involved in various stages of endodontic as well as peri-apical infections promotes bacterial colonization and activation of host defence mechanisms. Among various virulence factors of gram positive bacteria, Lipoteichoic acid (LTA) which has pathogenic potential similar to that of gram-negative bacteria virulence factor Lipopolysaccharide,plays a key role in serious inflammatory responses ${ }^{1,2}$

Teichoic acids (TAs) are specific polymers present on gram-positive bacterial cell surfaces. The word "teichoic" originates from the Greek word teikhos, meaning "wall" . Wall-teichoic acid (WTA) are those Teichoic acids (TA's) that are attached to the cell wall, where as those attached to the cell membrane are called as lipoteichoic acid (LTA). Armstrong et al was the first person to observe WTA in 1958 from cell wall fractions of Lactobacillus plantarum, Bacillus subtilis, and Staphylococcus aureus ${ }^{4}$. LTAs were identified by Kelemen et al. in 1961 in cell membrane fractions ${ }^{5}$.

LTA structure and function varies across different gram positive bacterial species. The back bone of TA's are made of polymers consisting of repeating polyolphosphate units. The backbone of LTA's are composed of poly-glycerol phosphate, and the WTA backbone are composed of poly-ribitol phosphate ${ }^{6}$. LTA are amphiphilic polysaccharides covalently linked to a terminal glycolipid moiety; and are released either during bacterial multiplication or after bacteriolysis caused by beta-lactam antibiotics or bactericidal cationic peptides, phospholipase $A$, cathepsins etc., ${ }^{1,7}$. LTA released after cell lysis binds specifically to target cells CD14 and to Toll-like receptors or binds non-specifically to membrane phospholipids. LTA bound to target cells causes activation of complement system and release of proinflammatory cytokines, reactive oxygen and nitrogen species from neutrophils and macrophages leading to indirect tissue injury ${ }^{7}$. The role of LTA in cell-to-cell interactions in biofilms, its role in causing cell envelope homeostasis were studied using the LTA deficient strain of Streptococcus gordonii ${ }^{8}$. LTA synthase(LtaS) enzyme inhibitor was found to prevent the growth of gram-positive antibiotic-resistant S. aureus in mice mode ${ }^{9}$

The present review attempts to summarize the immunostimulatory potential of gram-positive bacterial component LTA in causing endodontic infection and use of potential therapeutic agents against LTA inflammatory action.

\section{Data source}

A review of literature was performed by electronic search of Pubmed and Google Scholar for publications on LTA studies carried out mainly in relation to field of dentistry. Literature search was done using the key words - Lipoteichoic acid, endodontic infection, Enterococcus faecalis lipoteichoic acid, and endodontic medicaments. Articles were chosen using the following inclusion criteria: articles published in English language from 1953- January 2020, studies related to LTA role in endodontic infection were considered mainly. A total of 60 articles related to the field of Endodontics involved in studying the role of LTA in activating immune system in root canal and periapical infection were considered. Structure and role of LTA of those bacteria not exhibiting a potential role in endodontic infection were not considered.

Role of lipoteichoic Acid in Causing Tissue Injury Literature search shows that LTA can activate classic and alternative pathways of the complement system, activaties macrophages to release inflammatory mediators and causes several tissue alterations including bone resorption ${ }^{10}$. Various in vitro studies have shown variations in immunostimulatory potential of LTA and this variations can be avoided by more gentle high purification process of LTA extraction which can maintain the immunostimulatory potential ${ }^{11}$. LTA isolated from $E$. faecalis stimulates leukocytes to release inflammatory mediators like tumor 
necrosis factor alpha (TNF- $\alpha$ ), nitric oxide (NO) and chemokines through the Toll-likereceptor2 (TLR2) signaling pathway in macrophages. It also has role in biofilm formation that enables bacteria to survive in adverse environments ${ }^{12,13}$. Study carried out by Costa Junior et al has shown that Streptococcus faecalis LTA provoked a chronic inflammatory tissue reaction and can contribute to formation of a periapical lesion ${ }^{10}$.

LTA activates innate immune system which acts by detection of pathogens through specialized pattern recognition receptors like RIG-I-like receptor, the toll-like receptor family (TLR), and the nucleotide binding oligomerization domain (NOD)-like receptor (NLR) present in the cell membrane of immune and non-immune cells ${ }^{14}$. Among the pattern recognition receptors, TLR are key participants. Both LPS and LTA binds to $C D 14$ receptor and activates signalling by TLR and induces release of proinflammatory cytokines such as Tumor Necrosis Factor alpha (TNF- $\alpha$ ), Interleukin-1 (IL-1), Interleukin8 (IL-8), Interleukin-6, and anti-inflammatory cytokines like interleukin-10 and interleukin-12 $2^{2,15}$. Studies have also shown that LTA causes release of prostaglandin E2 (PGE2), lysosomal enzymes and the generation of superoxide anion. These factors which have tissue-damaging (TNF- $\alpha$, IL-1beta, IL-6, PGE2, lysosomal enzyme, superoxide anion) have been detected in periapical samples of patients with root canal infection ${ }^{12,16,17}$.

TNF- $\alpha$ is the most abundant cytokine and first bone resorptive mediator that has been detected in endodontic infection ${ }^{18}$. TNF- $\alpha$ induces osteoclast activation and also stimulates secretion of proteolytic enzymes and matrixmetallo proteinases(MMP), which are responsible for destroying extra cellular matrix of the bone tissue $^{19,20}$. In an experimental study conducted by Wang et al using clinical strain of E. faecalis, the authors concluded that $E$. faecalis LTA promoted pro-inflammatory cytokine TNF- $\alpha$ expression in macrophages by activating p38 Mitogenactivated protein kinases and nuclear factor kappa B (NF-KB) signalling pathways, but did not affect anti-inflammatory cytokines expression 21. Transcription factor NF- $K B$ is involved in cytokine synthesis and TLRs activate the effector phase of the innate immune response mainly through NF- $\kappa$ B pathway. In response to microbial infection,NLRP3 inflammasome that plays a key role in innate immunity, mediates caspase-1 activation which is essential for cleavage of proIL-1 $\beta$ and pro-IL-18 into their active mature form.

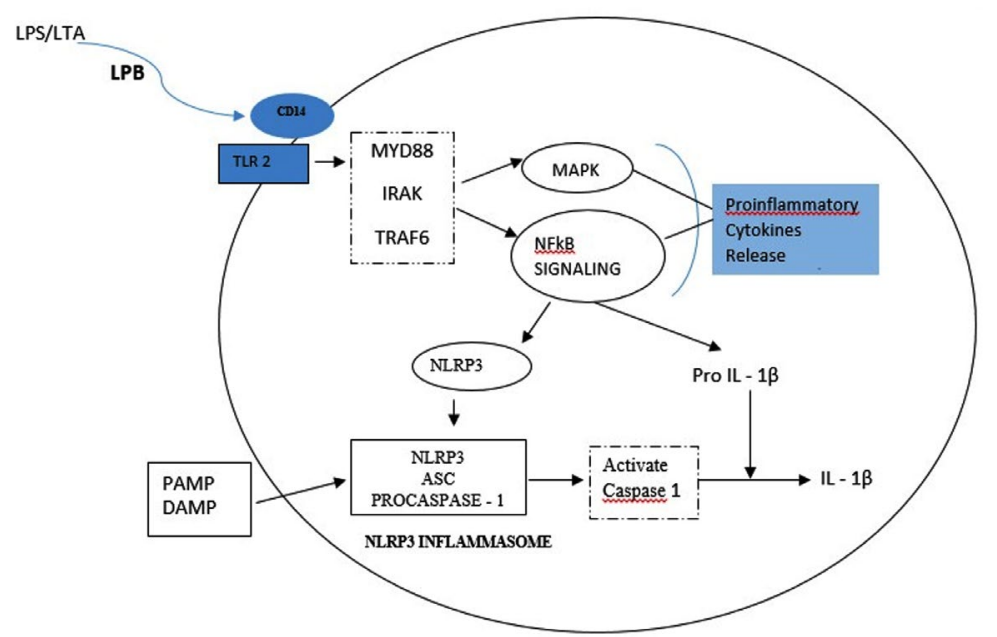

Fig. 1. LTA binds to CD14 receptor and are subsequently presented to Toll - like receptor (TLR). This stimulates the formation of a signalling complex that involves MyD88, IL-1receptor associated kinase (IRAK), and tumour necrosis factor receptor-associated factor 6 (TRAF 6). Signalling of this complex leads to activation of pathways that regulate NFKB nuclear factor - KB; MAP: mitogen - activating protein leading to release of pro inflammatory cytokines. A primary Signalling pathway through NFKB causes expression of NLRP3 and pro-IL-1 3 . A second signal PAMPs (Pathogen-Associated Molecular Patterns) and DAMPs (Danger-Associated Molecular Pattern) activates NLRP3 inflammasome assembly that leads to activation of caspase- 1 and the cleavage and secretion of active IL-1 $3 .{ }^{14,22}$ 
A study carried out by Wang et al revealed that during periapical periodontitis, E. faecalis LTA directly induces NLRP3 inflammasome expression associated with the activation of the NF-KB signalling pathway, activating high expression of caspase-1and release of IL- $1 \beta$, consequently leading to periapical inflammation ${ }^{14}$ (Fig - 1).

Konrad Tietze et al in their study found that gram-negative species produced high levels of TNF- $\alpha$, while gram-positive species released higher quantities of the chemokine, IL- 8 .This difference might be due to the reason that gram negative and gram positive bacteria activates different TLRs ${ }^{23}$. TLR-2 appears to be the primary mediator of the innate immune response to LTAs from various gram-positive bacteria ${ }^{24}$.

Experimental study carried out by Baik et al indicates that highly pure and structurally intact E. faecalis LTA stimulates TLR2 preferentially rather than TLR4. Predominant stimulation of TLR2 by gram-positive bacteria can also be influenced by the method of bacterial killing, such as heat killing or ethanol killing, since heat killing process can denature and inactivate proteinaceous antigens capable of stimulating TLR4. Studies have also stated that heat-killed $E$. faecalis has a unique feature that it was able to stimulate both TLR2 and TLR4, possibly because E. faecalis may possess heat-stable constituents which has the potential to stimulate TLR4 ${ }^{25}$. Despite the stimulation of both TLR2 and TLR4 by E. faecalis, TLR2 is more important than TLR4 in the dentinal inflammation because numerous TLR2-positive macrophages were detected, and the expression of TLR2 rather than TLR4 was greatly induced in inflamed dental pulp tissues ${ }^{25,26}$.

E.faecalis LTA increased macrophages autophagy which can have potential effect on process of periapical infection ${ }^{27}$. E. faecalis LTA inhibits osteoclast differentiation from bone marrow derived macrophages through TLR2 signalling pathway, and also causes the macrophages to maintain their phagocytic potential and their inflammatory capacities, possibly contributing to refractory apical periodontitis ${ }^{28}$. Similar results were obtained in another study where $E$. faecalis LTA effectively inhibited osteoclastogenesis of bone marrow macrophages in the presence of RANK via the transcription factor RBP-J ${ }^{29}$. The receptor activator of nuclear factor-KB ligand (RANKL)/ osteoprotegerin (OPG) are the prime regulators in bone resorption and have main functional role in the differentiation, activation and survival of osteoclasts and osteoclast precursors. RANKL triggers osteoclastogenesis thereby increasing bone loss, whilst OPG has the opposing effect. The results of study carried out Zhao et al shows that E. faecalis LTA upregulated the ratio of RANKL/OPG in a dose-dependent way and that only a lower concentrations was required to stimulate RANK ${ }^{30}$.

Yaguang Tian et al assessed the role of E. faecalis LTA on the apoptosis of osteoblasts and stated that the apoptotic effect of $E$. faecalis LTA on osteoblast was in a dose-dependent manner and LTA can inhibit the proliferation of human osteoblast-like cells. Periapical lesion may have an epithelial lining which acts as a barrier preventing the host cells from getting affected by slowing down the invasion of bacteria and their products into the host tissues. E.faecalis LTA has the ability to disrupt this epithelial barrier and affect osteoblasts in the periapical region ${ }^{31}$.

LTA up-regulates the expression of TLR2 and it was found that TLR2 activated odontoblast exhibits a down regulation in its special function of dentin matrix synthesis and mineralization and in turn these TLR2 activated odontoblast orients its activity on initiating innate immune response by secreting chemokines. An increased amount of inflammatory chemokine CCL2 and CXCL10 was found in supernatants from LTA-stimulated odontoblasts ${ }^{32}$.

In the work done by Wang PL et al, the authors stated that Streptococcus mutans LTA causes apoptosis of human dental pulp cells which is important for initiation and progression of pulpitis ${ }^{33}$. LTA causes rapid rise of macrophage Vascular Endothelial Growth Factor (VEGF) expression that may lead to pulpal necrosis or even to the prolonged maintenance of the inflammatory reaction after root canal therapy. In the experimental study carried out using Streptococcus mutans LTA, it was found that very low concentrations of LTA itself can stimulate macrophages in the dental pulp to secrete VEGF. VEGF is a strong inducer of angiogenesis and also increases vascular permeability. VEGF expression in dental pulp can cause increased intrapulpal pressure and pain ${ }^{34}$. 
In a study carried out to evaluate time dependent expression of complement $5 \mathrm{a}$ and its receptor C5aR, it was found that LTA at a concentration of $1 \mu \mathrm{g} / \mathrm{ml}$ stimulates human dental pulp cells to activate the expression of $\mathrm{C} 5 \mathrm{a}$ which was highest at 48 hours. Following initiation of inflammation, complementary protein fragments such as C3a, C4a and C5a are formed which causes degranulation of basophils/mast cells and release high amount of histamine, serotonin and vascular active mediators. The study also states that with in the timeline considered, the proinflammatory effect of LTA is stronger which is indicated by higher expression of IL- 6 by LTA than does LPS ${ }^{35}$.

Streptococcus mutans LTA were found to have high affinity for hydroxyapatite, suggesting LTA's significant role in colonization of gram positive bacteria on dentinal surfaces ${ }^{36}$.

The viable but nonculturable state is a survival mechanism adopted by bacteria when exposed to adverse environmental conditions and LTA has its role during this period as it was found that E.faecalis LTA quantity was doubled during this state $^{37}$. During this state bacteria loses its growth potential but retains its viability and pathogenicity and start its regular division upon restoration of normal growth conditions.

Werner Fischer defined polymeric chains associated with bacterial membranes using the generic term "amphiphile" and stated five structural types of LTA $(I-V)^{38}$. In the structure of E.faecalis LTA the hydrophilic chains are repetitious 1,3-polyglycerol phosphates and the C-2 position of the glycerol residues are nonstoichiometrically substituted with D-alanine and kojibiose residues ${ }^{39}$. E. faecalis LTA belongs to classical Type 1 LTA whose structure shows D-alanine esters substitute and this confer positive charges onto an otherwise negatively-charged polymer. Study carried out by Fabretti et al shows that LTA has a potential functional role in biofilm formation of $E$. faecalis, and an anti-LTA antibody affects biofilm formation. The effect of $D$-alanine esters of teichoic acids on biofilm formation and adhesions have also been evaluated ${ }^{40}$. The dlt operon, which causes expression of the protein that incorporates $D$ -alanine into LTA, consists of 4 genes: dltA, dltB, $\mathrm{dltC}$ and dltD and inactivation of genes within dit operon leads to total absence or decrease in $d$-alanine esters. The presence of D-alanine seem to be closely related to the inflammatory potential of LTA and absence of $D$-alanine residue affects the virulence of E.faecalis. D-alanylated LTA has the potential to restrict the action of autolysins and cell wall active antibiotics, and this helps bacterial envelop integrity to be maintained. Similar results were obtained by Poyart et al where a mutant strain of Streptococcus agalactiae which was lacking D-Alanine moiety on the LTA,exhibited a decrease in its virulence and was easily killed by macro phages and neutrophils ${ }^{41}$. Cetyltrimethylammonium bromide and chlorhexidine showed improved antimicrobial action on lactobacillus casei in which the gene dlt D was inactivated ${ }^{42}$.

Bovine mammary epithelial cells when stimulated with LTA resulted in upregulation of differentially expressed genes IL-8, CXCL6, CCL2 and IL- $1 \alpha$, proving that LTA play a role in initial inflammatory response ${ }^{43}$. LTA from pathogenic and non-pathogenic bacteria differ in their immunostimulatory abilities. LTA from pathogenic bacteria such as S.aureus induces production of NO and TNF $\alpha$ with effective TLR2 activation, whereas LTA from Lactobacillus plantarum suppresses TLR2 activation by attenuating Pam2CSK4 ligand induced IL-8 production in epithelial cells ${ }^{44,45}$. Considering these findings, it can be considered that LTA has a potential role in resistance against adverse conditions and also against root canal medicaments.

Therapeutic Medicaments in Endodontics to Neutralise Pathological Lipoteichoic Acid Activity

Antimicrobial agents are usually preferred for bacterial infection, but they are often not enough to obtain good treatment outcome, since there are only few effective endotoxinneutralizing agents. The overproduction of inflammatory mediators by pathogenic bacteria can be decreased by using agents that target LPS and LTA. Studies are being carried out with various endodontic medicaments that can bind to and sequester LTA and prevent excessive inflammatory response. Table 1 gives an insight about various studies carried out to test effectiveness of various medicaments on LTA in dentistry.

Stannous fluoride has clinical gingivitis benefits as it decreases the pathogenicity of plaque bio films by blocking reactivity of LPS and LTA ligands with tissue receptors that are involved 
Table 1. Summary of studies carried out to evaluate LTA neutralizing action by various therapeutic agents used

\begin{tabular}{ll}
\hline Authors \& Year & Study Purpose \\
\hline MatejaZorko et al 2008 [46] & $\begin{array}{l}\text { Study evaluated the effect of } \\
\text { alexidine and chlorhexidine } \\
\text { on LTA and LPS }\end{array}$ \\
& \\
Jung EunBaik et al 2008b [47] & $\begin{array}{l}\text { Study was carried out to evaluate } \\
\text { the role of calcium hydroxide in } \\
\text { inactivating E. faecalis LTA }\end{array}$
\end{tabular}

Jin-Kyung Lee et al 2009 [48]

Jung EunBaik et al 2011 [49]

Sung-Hoon Lee et al 2012 [50]
Study evaluated if Chlorhexidine decreases the inflammatory potential of Enterococcus faecalis and its virulence factor, Lipoteichoic acid

Study was carried out to evaluate if calcium hydroxide deacylates E. faecalis LTA resulting in the loss of immunostimulating activity

Study was carried out to investigate the antimicrobial and neutralizing effects of 4 Human $\beta$-Defensins $(H \beta D) 1,2,3,4)(H \beta D$ - cationic antimicrobial Peptides that plays a role in protecting the host against infectious microbes) on induction of proinflammatory cytokines by E. faecalis and E. faecalis LTA.

\section{Conclustion}

Study result showed that chlorhexidine had slightly better LPS neutralization action, while both chlorhexidine and alexidine showed similar LTAneutralizing activity. Both chlorhexidine and alexidine had the potential to neutralize immunostimulatory cell-wall components released after bactericidal activity of antibiotics.

The result of the study shows that inflammatory activity of LTA is inactivated by calcium hydroxide by affecting its TLR2-stimulating activity resulting in reduced TNF- $\alpha$ expression. Chlorhexidine can inactivate LTA of $E$. faecalis leading to a decrease in inflammatory responses.

To totally neutralize LTA's inflammatory potential, chlorhexidine greater than $0.2 \%$ concentration was needed for 24 hours of treatment time and with $2 \%$ chlorhexidine a treatment time more than 6 hours was needed. Results of study states that calcium hydroxide could deacylate the gycolipid moiety of E.faecalis LTA, leading to impairment of immunostimulating activity.

It was found that $\mathrm{H} \beta \mathrm{D}$ s had an antibacterial effect against E. faecalis, All tested H $\beta D$ s inhibited $E$. faecalis LTA induced expression of proinflammatory cytokine and intercellular adhesion molecule 1 $\mathrm{H} \beta \mathrm{D}-3$ showed strongest bactericidal and neutralizing effect against $E$. faecalis and E. faecalis LTA. 


$\begin{array}{lll}\text { Sun Woong Hong et al } 2016 \text { [51] } & \begin{array}{l}\text { Study was carried out to } \\ \text { investigate whether } \mathrm{NaOCl} \\ \text { inactivates E.faecalis LTA. }\end{array} & \begin{array}{l}\text { Treatment of E.faecalis LTA with } \\ \text { NaOCl causes a reduced } \\ \text { production of inflammatory } \\ \text { mediators by LTA, as NaOCl } \\ \text { deacylates the glycolipid moiety } \\ \text { of E.faecalis LTA structure. }\end{array} \\ \text { Wei Yin et al } 2020[52] & \begin{array}{l}\text { Results showed that LTA induced } \\ \text { activation of NLRP3 inflammasome } \\ \text { in mouse macrophages was }\end{array} \\ & \begin{array}{l}\text { Study evaluated effect of } \\ \text { dioscin, an anti-oxidative } \\ \text { stress drug, on the }\end{array} & \begin{array}{l}\text { inhited by dioscin } \\ \text { induced activation of } \\ \text { E. faecalis originated LTA }\end{array} \\ & \begin{array}{l}\text { NLR3 inflammasome in } \\ \text { mouse Macrophages }\end{array}\end{array}$

in inflammation ${ }^{53,54}$. Studies were carried out to evaluate the reactivity of LPS and LTA with cationically charged cetylpyridinium chloride and stannus fluoride and the result showed that both cetylpyridinium chloride and stannus salts interfered with LPS and LTA binding to cellular $T^{5}{ }^{55}$

\section{Effect of LTA against Bacterial biofilm}

It is stated that LTA is involved in biofilm formation. However, LTA of few bacterial species like Lactobacillus plantarum which is a known probiotic agent in oral health has shown to have inhibitory effect on Staphylococcus aureus biofilm formation ${ }^{56}$. Biofilm formation of multispecies bacteria was decreased in dose dependent way by Lactobacillus plantarum LTA (Lp.LTA). Intracanal medicaments efficacy in removal of preformed multispecies biofilm was aided by Lactobacillus plantarum LTA ${ }^{57}$. Disruption of E. faecalis biofilms on dentin blocks were enhanced when dentin blocks were pretreated with Lp. LTA and then followed by endodontic medicaments use, than when using Lp. LTA or medicament alone, or when using both at the same time ${ }^{58}$. Among various Lactobacillus species studied,Lp. LTA has a potential biofilm blocking effect and this inhibition is seen not only on E. faecalis biofilm but also on other bacterial biofilms ${ }^{59}$. Compound 1771 that modulates LTA metabolism inhibited Streptococcus mutants biofilm formation and this effect was more pronounced when it was used along with myrcitin and fluoride. ${ }^{60}$

\section{CONCLUSION}

LPS is the commonly investigated bacterial virulence factor but taking into account that endodontic infections are polymicrobial in nature studies can also be focused on influence of other bacterial virulence factors. Little is known in the endodontic literature regarding the LTA of grampositive bacteria in teeth with regard to its role to clinical finding. With this consideration further research related to LTA structure of common gram positive endodontic pathogen which may aid in development of methods to neutralize LTA pathological potential. Considering factors like LTA extraction method, contamination of LTA used in studies by endotoxins and structural deterioration during in vitro studies, further studies correlating role of LTA in clinical symptoms are to be carried out. The immunostimulatory effect of LTA in causing infection merit further investigation by studies to evaluate precise role of LTA in causing periapical infection, ways for elimination of LTA by developing novel therapeutic agents targeting LTA, and relationship of LTA contents and clinical symptoms for a better treatment outcome.

\section{ACKNOWLEDGMENTS}

None.

\section{CONFLICT OF INTEREST}

The authors declare that there is no conflict of interest. 


\section{AUTHORS' CONTRIBUTION}

VSR Conceptualized the study. VSR, MR, $A V$ did the data curation. BRM visualized the study. MR and VS provided the necessary resources and did the supervision. VSR administered the project. VSR wrote the original draft. VSR, BRM and AV wrote the manucript. $\mathrm{KV}$ revised the manuscript. BRM, AV and KV reviewed and edited the final manuscript.

\section{FUNDING}

\section{None}

\section{DATA AVAILABILITY}

The datasets generated analysed during the current study are available from the corresponding author on reasonable request.

\section{ETHICS STATEMENT}

This article does not contain any studies with human participants or animals performed by any of the authors.

\section{REFERENCES}

1. Barbosa-Ribeiro $M$, De-Jesus-Soares $A$, Zaia AA, Ferraz CC, Almeida JF, Gomes BP. Quantification of lipoteichoic acid contents and cultivable bacteria at the different phases of the endodontic retreatment. J. Endod, 2016; 42: 552-556. doi: 10.1016/j.joen.2016.01.002

2. Parolia A, Gee LS, Porto I, Mohan M. Role of cytokines, endotoxins (LPS), and lipoteichoic acid (LTA) in endodontic infection. J Dent Oral Disord Ther 2014; 2(4):1-5. doi:10.15226/jdodt.2014.00132

3. Kleerebezem M, Hols P, Bernard E, et al. The extracellular biology of the lactobacilli. FEMS microbiology reviews. 2010;34(2):199-230. doi:10.1111/j.15746976.2009.00208.x

4. Armstrong JJ, Baddiley J, Buchanan JG, Carss B, Greenberg GR. 882. Isolation and structure of ribitol phosphate derivatives (teichoic acids) from bacterial cell walls. J. Chem. Soc(Resumed). 1958:4344-4354. doi: 10.1039/jr9580004344

5 Kelemen MV, Baddiley J. Structure of the intracellular glycerol teichoic acid from Lactobacillus casei ATCC 7469. Biochem. J. 1961;80(2):246-254. doi: 10.1042/ bj0800246

6. Araki Y, Ito E. Linkage units in cell walls of gram-positive bacteria. Crit Rev Microbiol. 1989;17(2):121-135. doi:10.3109/10408418909105745

7. Ginsburg I. Role of lipoteichoic acid in infection and inflammation. Lancet Infect Dis. 2002;2(3): 171-179. doi: 10.1016/S1473-3099(02)00226-8

8. Lima BP, Kho K, Nairn BL, et al. Streptococcus gordonii type I lipoteichoic acid contributes to surface protein biogenesis. Msphere. 2019;4(6)e00814-19. doi:
10.1128/mSphere.00814-19

9. Richter SG, Elli D, Kim HK, et al. Small molecule inhibitor of lipoteichoic acid synthesis is an antibiotic for Gram-positive bacteria. Proc Natl Acad Sci. 2013 ; 110(9):3531-3536. doi: 10.1073/pnas. 1217337110

10. Costa Junior ED, Souza-Filho FJ, Barbosa SV. Tissue reactions to a component of root canal system bacteria: lipoteichoic acid. Braz. Dent. J. 2003;14:9598. doi: 10.1590/S0103-64402003000200004

11. Hermann C, Spreitzer I, Schröder NW, et al. Cytokine induction by purified lipoteichoic acids from various bacterial species-Role of LBP, sCD14, CD14 and failure to induce IL 12 and subsequent IFN $\gamma$ release. Eur. J. Immunol. 2002;32(2):541-551. doi: 10.1002/1521-4141(200202)32:2<541::AIDIMMU541>3.0.CO;2-P

12. Card GL, Jasuja RR, Gustafson GL. Activation of arachidonic acid metabolism in mouse macrophages by bacterial amphiphiles. J. Leukoc. Biol. 1994 ;56(6):723-728. doi: 10.1002/jlb.56.6.723

13. Kayaoglu G, Ørstavik D. Virulence factors of Enterococcus faecalis: relationship to endodontic disease. Crit Rev Oral Biol Med. 2004;15(5):308-320. doi: 10.1177/154411130401500506

14. Wang L, Jin H, Ye D, et al. Enterococcus faecalislipoteichoic acid-induced NLRP3 inflammasome via the activation of the nuclear factor kappa $B$ pathway. J. Endod. 2016;42(7):1093-1100.doi: 10.1016/j.joen.2016.04.018

15. Cleveland MG, Gorham JD, Murphy TL, Tuomanen E, Murphy KM. Lipoteichoic acid preparations of gram-positive bacteria induce interleukin-12 through a CD14-dependent pathway. Infect. Immun. 1996;64(6):1906-1912. doi: 10.1128/IAI.64.6.19061912.1996

16. Harrop PJ, O'Grady RL, Knox KW, Wicken AJ. Stimulation of lysosomal enzyme release from macrophages by lipoteichoic acid. J. Periodontal Res. 1980;15(5):492501. doi: 10.1111/j.1600-0765.1980.tb00307.x

17. Levy $R$, Kotb $M$, Nagauker $O$, et al. Stimulation of oxidative burst in human monocytes by lipoteichoic acids. Infect. Immun. 1990;58(2):566-568. doi: 10.1128/IAI.58.2.566-568.1990

18. Marinho AC, Martinho FC, Leite FR, Nascimento GG, Gomes BP. Proinflammatory activity of primarily infected endodontic content against macrophages after different phases of the root canal therapy. J. Endod. 2015;41(6):817-823. doi: 10.1016/j. joen.2015.01.017

19. Chang YC, Yang SF, Huang FM, Tai KW, Hsieh YS. Induction of tissue plasminogen activator gene expression by proinflammatory cytokines in human pulp and gingival fibroblasts. J. Endod. $2003 ; 29(2)$ :114117. doi: 10.1097/00004770-200302000-00007

20. Pezelj-Ribaric S, Magašic K, Prpic J, Miletic I, Karlovic Z. Tumor necrosis factor-alpha in peripical tissue exudates of teeth with apical periodontitis. Mediat Inflamm. 2007. doi: 10.1155/2007/69416

21. Wang S, Liu KU, Seneviratne CJ, et al. Lipoteichoic acid from an Enterococcus faecalis clinical strain promotes TNF- $\alpha$ expression through the NF- $\kappa B$ and p38 MAPK signaling pathways in differentiated THP-1 
macrophages. Biomed. Rep. 2015;3(5):697-702. doi: 10.3892/br.2015.495

22. Tõzsér J, Benkõ S. Natural compounds as regulators of NLRP3 inflammasome-mediated IL-1 $\beta$ production. Mediat Inflamm. 2016. doi: 10.1155/2016/5460302

23. Tietze K, Dalpke A, Morath S, Mutters R, Heeg K, Nonnenmacher C. Differences in innate immune responses upon stimulation with gram positive and gram negative bacteria J. Periodontal Res. $2006 ; 41(5): 447-454$. doi: $10.1111 /$ j.16000765.2006.00890.x

24. Long EM, Millen B, Kubes P, Robbins SM. Lipoteichoic acid induces unique inflammatory responses when compared to other toll-like receptor 2 ligands. PloS one. 2009;4(5):e5601. doi: 10.1371/journal. pone.0005601

25. Baik JE, Ryu YH, Han JY, et al. Lipoteichoic acid partially contributes to the inflammatory responses to Enterococcus faecalis. J. Endod. 2008 ;34(8):975-982. doi: 10.1016/j.joen.2008.05.005

26. Mutoh N, Tani-Ishii N, Tsukinoki K, Chieda K, Watanabe K. Expression of toll-like receptor 2 and 4 in dental pulp. J. Endod. 2007;33(10):1183-1186. doi: 10.1016/j. joen.2007.05.018

27. Lin D, Gao Y, Zhao L, Chen Y, An S, Peng Z. Enterococcus faecalislipoteichoic acid regulates macrophages autophagy via PI3K/Akt/mTOR pathway. Biochem. Biophys. Res. Commun. 2018;498(4):1028-1036. doi: 10.1016/j.bbrc.2018.03.109

28. Yang J, Park OJ, Kim J, Baik JE, Yun CH, Han SH. Lipoteichoic acid of Enterococcus faecalis inhibits the differentiation of macrophages into osteoclasts. J. Endod. 2016;42(4):570-574. doi: 10.1016/j. joen.2016.01.012

29. Wang S, Heng BC, Qiu S, et al. Lipoteichoic acid of Enterococcus faecalis inhibits osteoclastogenesis via transcription factor RBP-J. Innate Immun. 2019;25(1):13-21. doi: $10.1177 / 1753425918812646$

30. Zhao L, Chen J, Cheng L, et al. Effects of Enterococcus faecalis lipoteichoic acid on receptor activator of nuclear factor $\kappa B$ ligand and osteoprotegerin expression in periodontal ligament fibroblasts. Int Endod J. 2014;47(2):163-172. doi: 10.1111/ iej.12127

31. Tian $Y$, Zhang $X$, Zhang $K$, et al. Effect of Enterococcus faecalis lipoteichoic acid on apoptosis in human osteoblast-like cells. J. Endod. $2013 ; 39(5): 632-637$. doi: 10.1016/j.joen.2012.12.019

32. Durand SH, Flacher V, Roméas A, et al. Lipoteichoic acid increases TLR and functional chemokine expression while reducing dentin formation in in vitro differentiated human odontoblasts. The J. Immunol. 2006;176(5):2880-2887. doi: 10.4049/ jimmunol.176.5.2880

33. Wang PL, Shirasu S, Daito M, Ohura K. Streptococcus mutanslipoteichoic acid-induced apoptosis in cultured dental pulp cells from human deciduous teeth. Biochem. Biophys. Res. Commun. 2001;281(4):957961.doi: 10.1006/bbrc.2001.4451

34. Telles PD, Hanks CT, Machado MA, Nör JE. Lipoteichoic acid up-regulates VEGF expression in macrophages and pulp cells. J. Dent. Res. 2003;82(6):466-470. doi: 10.1177/154405910308200612

35. Liu M, Mu H, Peng W, et al. Time-dependent C5a and $\mathrm{C} 5 \mathrm{aR}$ expression in dental pulp cells following stimulation with LTA and LPS. Int J Mol Med. 2019; 44(3):823-834. doi: 10.3892/ijmm.2019.4246

36. Ciardi JE, Rölla G, Bowen WH, Reilly JA. Adsorption of Streptococcus mutanslipoteichoic acid to hydroxyapatite. Eur J Oral Sci. 1977;85(6):387-391. doi: 10.1111/j.1600-0722.1977.tb00570.x

37. Signoretto C, del Mar Lleò M, Tafi MC, Canepari P. Cell Wall Chemical Composition ofEnterococcusfaecalis in the Viable but Nonculturable State. Appl. Environ. Microbiol. 2000;66(5):1953-1959. doi: 10.1128/ AEM.66.5.1953-1959.2000

38. Schneewind O, Missiakas D. Lipoteichoic acids, phosphate-containing polymers in the envelope of gram-positive bacteria. J. Bacteriol. 2014 ;196(6):11331142. doi: 10.1128/JB.01155-13

39. Theilacker C, Kaczynski Z, Kropec A, et al. Opsonic antibodies to Enterococcus faecalis strain 12030 are directed against lipoteichoic acid. Infect. Immun. 2006 ;74(10):5703-5712.doi: 10.1128/IAI.00570-06

40. Fabretti F, Theilacker C, Baldassarri L, et al. Alanine esters of enterococcallipoteichoic acid play a role in biofilm formation and resistance to antimicrobial peptides. Infect. Immun. 2006;74(7):4164-4171. doi: 10.1128/IAI.00111-06

41. Poyart C, Pellegrini E, Marceau M, et al. Attenuated virulence of Streptococcus agalactiae deficient in $D$ alanyl lipoteichoic acid is due to an increased susceptibility to defensins and phagocytic cells. Mol. Microbiol. 2003;49(6):1615-1625. doi: 10.1046/j.13652958.2003.03655.x

42. Debabov DV, Kiriukhin MY, Neuhaus FC. Biosynthesis of Lipoteichoic Acid inLactobacillusrhamnosus: Role of DItDind-Alanylation. J. Bacteriol. 2000;182(10):28552864. doi: 10.1128/JB.182.10.2855-2864.2000

43. Kiku $Y$, Nagasawa $Y$, Tanabe $F$, et al. The cell wall component lipoteichoic acid of Staphylococcus aureus induces chemokine gene expression in bovine mammary epithelial cells. J Vet Med Sci. 2016:79(9):1505-1510. doi: 10.1292/jvms.150706

44. Ryu YH, Baik JE, Yang JS, et al. Differential immunostimulatory effects of Gram-positive bacteria due to their lipoteichoic acids. Int. Immunopharmacol. 2009 ;9(1):127-133. doi: 10.1016/j.intimp.2008.10.014

45. Noh SY, Kang SS, Yun CH, Han SH. Lipoteichoic acid from Lactobacillus plantarum inhibits Pam2CSK4-induced IL-8 production in human intestinal epithelial cells. Mol. Immunol. 2015;64(1):183-189. doi: 10.1016/j. molimm.2014.11.014

46. Zorko M, Jerala R. Alexidine and chlorhexidine bind to lipopolysaccharide and lipoteichoic acid and prevent cell activation by antibiotics. J Antimicrob Chemother. 2008 ;62(4):730-737. doi: 10.1093/jac/dkn270

47. Baik JE, Kum KY, Yun $\mathrm{CH}$, et al. Calcium hydroxide inactivates lipoteichoic acid from Enterococcus 
faecalis. J. Endod. 2008 ;34(11):1355-1359.doi: 10.1016/j.joen.2008.08.014

48. Lee JK, Baik JE, Yun CH, et al. Chlorhexidine gluconate attenuates the ability of lipoteichoic acid from Enterococcus faecalis to stimulate toll-like receptor 2. J. Endod. 2009;35(2):212-215. doi: $10.1016 / \mathrm{j}$ joen.2008.10.018

49. Baik JE, Jang KS, Kang SS, et al. Calcium hydroxide inactivates lipoteichoic acid from Enterococcus faecalis through deacylation of the lipid moiety. J. Endod. $2011 ; 37(2): 191-196$. doi: 10.1016/j.joen.2010.11.007

50. Lee SH, Baek DH. Antibacterial and neutralizing effect of human $\beta$-defensins on Enterococcus faecalis and Enterococcus faecalis lipoteichoic acid. J. Endod. 2012 ; 38(3):351-356. doi: 10.1016/j. joen.2011.12.026

51. Hong SW, Baik JE, Kang SS, Kum KY, Yun $\mathrm{CH}$, Han $\mathrm{SH}$. Sodium hypochlorite inactivates lipoteichoic acid of Enterococcus faecalis by deacylation. J. Endod. 2016;42(10):1503-1508. doi: 10.1016/j. joen.2016.06.018

52. Yin W, Liu S, Dong M, et al. A New NLRP3 inflammasome inhibitor, dioscin, promotes osteogenesis. Small. 2020 ;16(1):1905977. doi: 10.1002/smll.201905977

53. Haught C, Xie S, Circello B, et al, White DJ. Lipopolysaccharide and Lipoteichoic Acid Virulence Deactivation by Stannous Fluoride. J Clin Dent. 2016;27(3):84-89.

54. Klukowska M, Haught JC, Xie S, et al. Clinical effects of stabilized stannous fluoride dentifrice in reducing plaque microbial virulence I: Microbiological and receptor cell findings. J Clin Dent.2017;28:1626.

55. Haught JC, Xie S, Circello B, et al. Lipopolysaccharide and lipoteichoic acid binding by antimicrobials used in oral care formulations. Am J Dent. 2016;29(6):328332.

56. Ahn KB, Baik JE, Yun CH, Han SH. Lipoteichoic acid inhibits Staphylococcus aureus biofilm formation. Front. Microbiol. 2018;9:327. doi: 10.3389/ fmicb.2018.00327

57. Kim AR, Ahn KB, Yun $\mathrm{CH}$, et al. Lactobacillus plantarum lipoteichoic acid inhibits oral multispecies biofilm. J. Endod. 2019 ;45(3):310-315. doi: 10.1016/j. joen.2018.12.007

58. Kim AR, Kang M, Yoo YJ, et al. Lactobacillus plantarum lipoteichoic acid disrupts mature Enterococcus faecalis biofilm. J. Microbiol. 2020;58(4)314-319.

59. Jung S, Park OJ, Kim AR, et al. Lipoteichoic acids of lactobacilli inhibit Enterococcus faecalis biofilm formation and disrupt the preformed biofilm. J. Microbiol. 2019 ;57(4):310-315. doi: 10.1007/s12275019-8538-4

60. Castillo Pedraza MC, de Oliveira Fratucelli ED, Ribeiro SM, Florez Salamanca EJ, da Silva Colin J, Klein MI. Modulation of Lipoteichoic Acids and Exopolysaccharides Prevents Streptococcus mutans Biofilm Accumulation. Molecules. 2020 ;25(9):2232. doi: $10.3390 /$ molecules 25092232 